\title{
Understanding the Sharing of Rival Information: A Simulation Approach
}

\author{
Yan Qu \\ College of Information Studies, University of Maryland, College Park \\ yanqu@umd.edu
}

\section{Jun Zhang}

School of Information, University of Michigan, Ann Arbor junzh@umich.edu

In this preliminary study, we proposed a mathematical information sharing model for rival information and simulated the information sharing behaviors in systems confronting the social dilemma. The simulations help us understand the factors that influence the performance of information sharing systems and may lead to new approaches that solve the social dilemma.

Introduction

The development of Web2.0 has seen a wide range of systems in which people create and share information directly with others, such as Wikipedia, Slashdot, and Yahoo Answers. Whether the users are willing to share information with others is a key factor of the success of these large-scale Web based collaboration systems or services. Many studies have attempted to explain why people voluntarily participate in these collaboration systems. Known theories include anticipated reciprocity, increased recognition, sense of efficacy, and sense of community [1].

An important characteristic of these systems is that the shared information is mostly "nonrival", which means the information value does not change no matter whether it is owned by one or thousands. However, there is also "rival" information, whose value decreases once it is shared with others, such as a new business idea, or a secret parking spot. The sharing of rival information is rare in these web communities because of the social dilemma [2][3]: a situation where an individual's interest conflicts with the group's interest. A better understanding of such phenomena may foster the sharing of rival information in future systems. 
In this preliminary study, we proposed a mathematical information sharing model for rival information and simulated the information sharing behaviors in systems confronting the social dilemma. The simulations help us understand the factors that influence the performance of information sharing systems and may lead to new approaches that solve the social dilemma.

\section{Information sharing model}

To analyze sharing trends in information-sharing systems confronting the social dilemma, an information sharing model was proposed:

1. There are two types of information: public information and private information. Public information is possessed by everyone in the group. Private information is possessed by just one person in the group.

2. A piece of information has similar value to all individuals. When a piece of information is private to an individual, its value is 1; when the owner shares it with others, its value decreases to $(1-\alpha)$.

3. In a group of $n$ individuals, the information flow of individual $i$ is shown in figure 1 .

$\alpha_{i}$ is valuable information that individual $i$ retrieves by himself; $b_{i j}$ is useful information he gets from others; $c i$ is information he shares with others.

Figure 1. Information flow

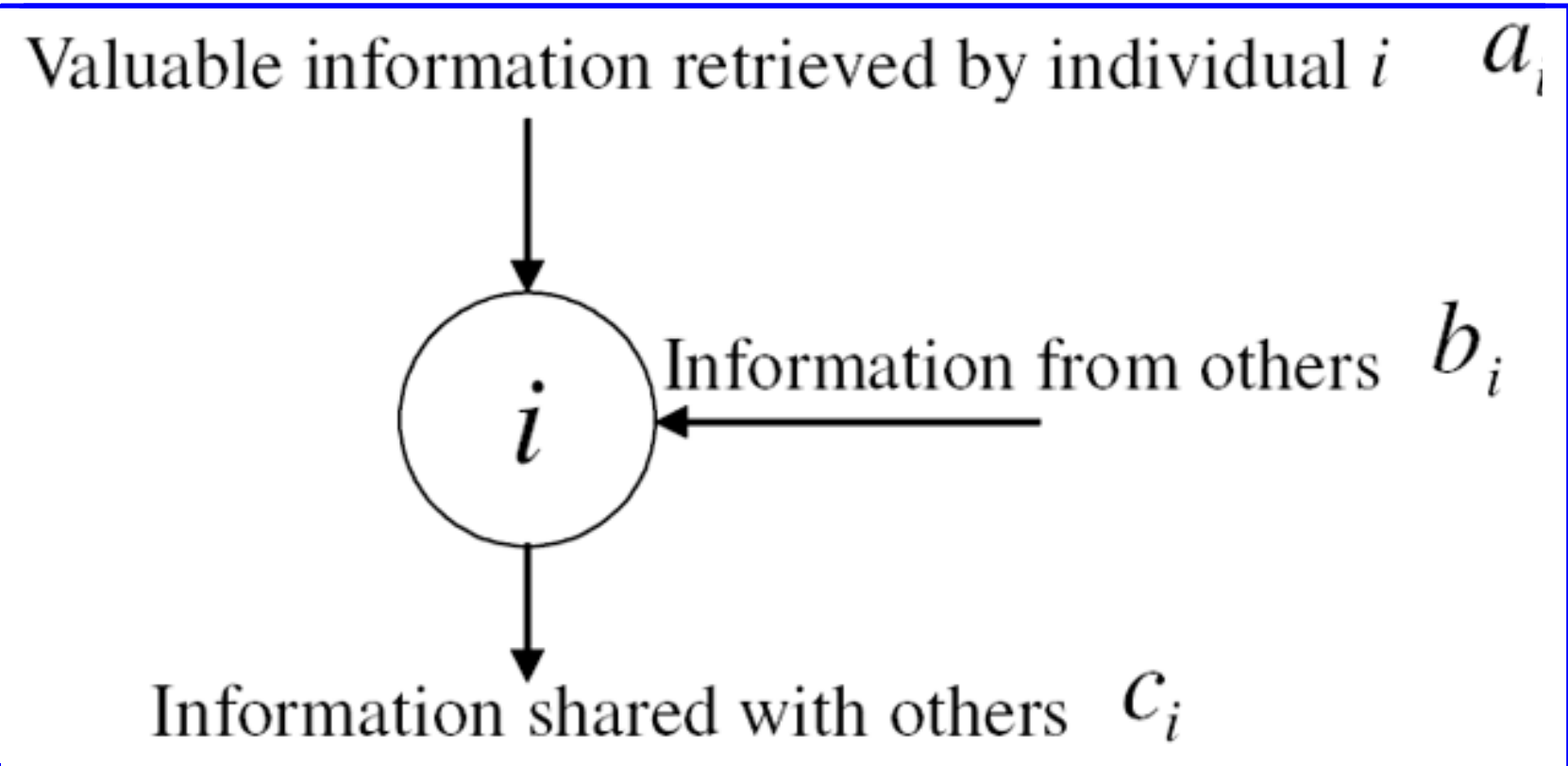

4. We introduce a variable $\beta i$ to represent an individual i's willingness to share information. The greater the $\beta i$ is, the more the individual $i$ is willing to share 
information with others. The Value of $\beta i$ is between 1 and 0.

$c i$ and $b_{i}$ can be calculated as follows:

$$
\begin{gathered}
c_{i}=\beta_{i} a_{i} \quad 0 \leq \beta_{i} \leq 1 \\
b_{i}=\sum_{j \neq i} c_{j}=\sum_{j \neq i} \beta_{j} a_{j}
\end{gathered}
$$

5. The payoff to an individual is the profit he derives from the group. The payoff to individual $i$ is:

$$
\begin{aligned}
& H_{i}=(1-\alpha) b_{i}-\alpha c_{i} \\
& =(1-\alpha) \sum_{j \neq i} \beta_{j} a_{j}-\alpha \beta_{i} a_{i}
\end{aligned}
$$

6. We assume that individuals always pursue maximum interests, thus they select actions that bring them profits and avoid actions that make them lose profits. Therefore, to pursue maximum interests, the individuals adjust their $\beta$ s according to the changes of their payoffs $H^{(k)}-H^{(k-1)}$ and the previous directions $\operatorname{dir}(k)$ in which they adjusted $\beta$ s:

$$
\beta_{i}^{(k+1)}=\beta_{i}^{(k)}+\operatorname{sign}\left(H_{i}^{(k)}-H_{i}^{(k-1)}\right) d i r_{i}^{(k)} \mu_{i}^{(k+1}
$$

Where $\mu_{i}(k+1)$ is the pace of the adjustment; $\eta_{i}(k+1)$ is a random variable representing uncertainties in the adjustments of $\beta i$.

\section{Analysis of sharing trends}

\section{Synchronous situation}

Firstly, we give the theoretical deduction in a situation where individuals adjust their $\beta \mathrm{s}$ synchronously. Three simplifications are adopted:

1. The amount of valuable information retrieved by any individual during any period is 
the same.

2. There are no uncertainties in adjustment.

3. Individuals adjust their $\beta$ s with uniform paces.

Under these simplifications, the change of payoff to individual $i$ is:

$$
\begin{aligned}
& \Delta H_{i}^{(k)}=H_{i}^{(k)}-H_{i}^{(k-1)} \\
& =\left((1-\alpha) b_{i}^{(k)}-\alpha c_{i}^{(k)}\right)-\left((1-\alpha) b_{i}^{(k-1)}-\alpha c_{i}^{(k-1)}\right) \\
& =\left((1-\alpha) \sum_{j \neq i}\left(\beta_{j}^{(k)}-\beta_{j}^{(k-1)}\right)-\alpha\left(\beta_{i}^{(k)}-\beta_{i}^{(k-1)}\right)\right) a(5) \\
& =\left(-\Delta \beta_{i}^{(k)}+(1-\alpha) \sum_{j} \Delta \beta_{j}^{(k)}\right) a
\end{aligned}
$$

For further analysis, equation (6) is given:

$$
\sum_{j} \Delta \beta_{j}^{(k)}=m_{i}^{(k)} \Delta \beta_{i}^{(k)}
$$

In this equation, the overall change of $\beta$ in the group is $m_{i}(k)$ times as much as $\Delta \beta_{i}(k)$. If we use $w^{(k)}$ to represent the number of individuals who increase their $\beta$ s and use $v(k)$ to represent the number of individuals who decrease their $\beta$, we have:

$$
\begin{gathered}
\left|m_{i}^{(k)}\right|=\left|w^{(k)}-v^{(k)}\right|<n \\
\Delta H_{i}^{(k)}=\left(-1+(1-\alpha) m_{i}^{(k)}\right) \Delta \beta_{i}^{(k)} a(8)
\end{gathered}
$$

According to equation (4) and (8), individual $i$ will increase his $\beta i$ when $m_{i}^{(k)}>\frac{1}{1-\alpha}$, and decrease his $\beta i$ when $m_{i}^{(k)}<\frac{1}{1-\alpha}$. This leads to the 
following deduction: If $\alpha>\frac{n-1}{}$, then there will not be trends toward sharing in n

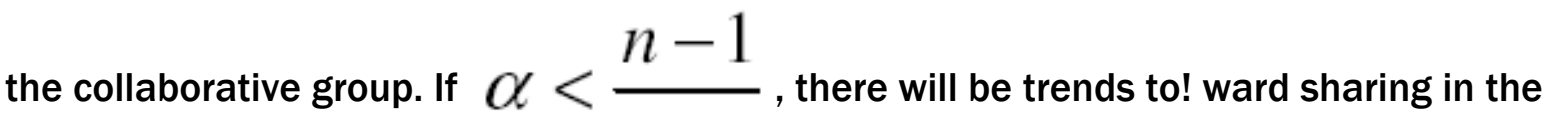
$n$ collaborative group.

Using some skillful transformations, we can remove these simplifications and have most of our deductions unchanged.

\section{Asynchronous situation}

In the deduction in 3.1, $m_{i}(k)$, the imbalance of the individuals' decisions which occurs when individuals adjust their behaviors simultaneously is the intrinsic impetus of trends toward sharing in the collaborative group. This imbalance will be weakened in asynchronous situations since few people act simultaneously. As a result, there will not be clear trends toward sharing in the asynchronous systems

\section{Simulation}

We present results of a simulation with a group of 50 individuals. In the simulation, each individual adjusted his cooperative degree $\beta$ to pursue the maximum interest. $\beta$ s took values in the interval of $(0,1)$ and were assigned random values at the beginning of the simulation. The distribution of $\beta$ s was used as our result after performing a thousand trials. The simulation was repeated for three different values of $\alpha(0.5,0.9,0.99)$.

Figure 2 shows the simulation results. The $x$-axis is people's willingness to share. The $y$-axis is the percentage of people. Each curve shows people's willingness to share under a certain $\alpha$ value. The results conform our theoretical analysis: there are obvious sharing $(\alpha=0.5,0.9)$ and unsharing $(\alpha=0.99)$ trends in the simplified synchronous situation while no obviously sharing trends in the asynchronous situation.

Figure 2. Distribution of $\beta$ s in different situations

(a) Synchronous situation 


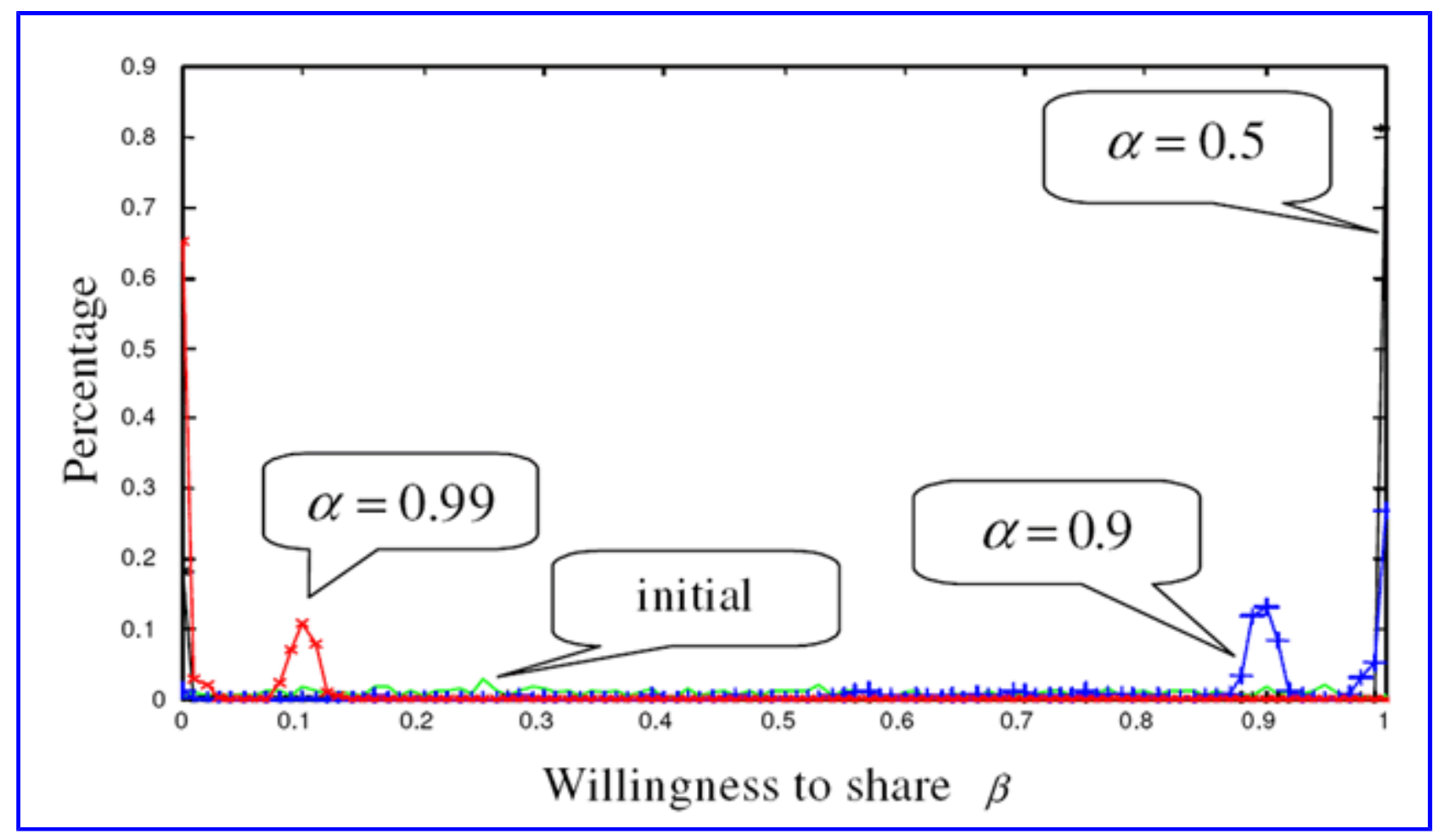

(b) Asynchronous situation

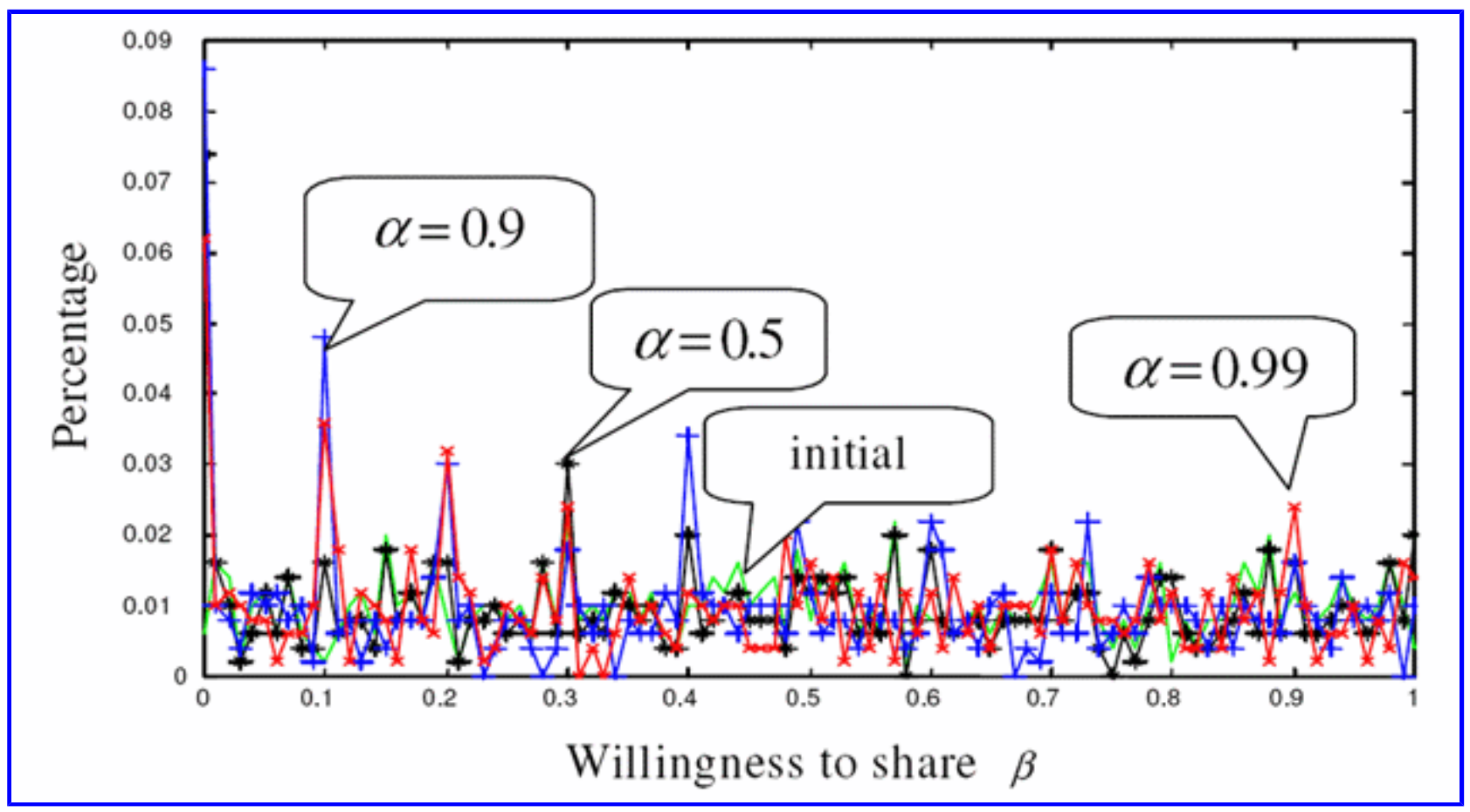

\section{Conclusion}

This poster presents an effort to understand the sharing of rival information in a group. The theoretical deduction and the simulations suggest that sharing trends may appear more easily in synchronous situations than asynchronous situations. In asynchronous situations, 
which is the case for most online information sharing systems, more incentive mechanism are needed to foster sharing of rival information. The current information sharing model is still preliminary. Future work will focus on refining the model with findings from empirical studies on information sharing behaviors.

\section{References}

Kollock, P. 1999. "The Economies of Online Cooperation: Gifts and Public Goods in Cyberspace," Communities in Cyberspace. Marc Smith and Peter Kollock (editors). London: Routledge.

Glance, N.S. and Huberman, B. A. 1994. The Dynamics of Social Dilemmas, Scientific American, 270, pp. 76-81.

Lichbach, M. I. 1996. The Cooperator's Dilemma. University of Michigan Press, Ann Arbor. 